\title{
Generation of optical needle and dark channel by tight focusing of radially polarized circular partially coherent beams
}

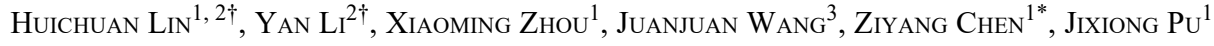 \\ ${ }^{1}$ Fujian Provincial Key Laboratory of Light Propagation and Transformation, \\ College of Information Science and Engineering, Huaqiao University, \\ Xiamen 361021, China \\ ${ }^{2}$ College of Physics and Information Engineering, Minnan Normal University, \\ Zhangzhou 363000, China \\ ${ }^{3}$ College of Information and Electronic Engineering, Liming Vocational University, \\ Quanzhou 362000, China \\ *Corresponding author: ziyang@hqu.edu.cn
}

\begin{abstract}
We theoretically investigate the tight focusing of radially polarized circular partially coherent (RPCPC) beams through a high numerical aperture objective. The sub-wavelength super-long optical needle and dark channel can be obtained near the focus, by engineering the source coherent length of the incident RPCPC beams. The length of the optical needle and the dark channel can be adjusted, and the obtained maximal lengths of the optical needle and the dark channel are both $22 \lambda$. The full width at half maximum of the optical needle and the dark channel are $0.6 \lambda$ and $0.48 \lambda$, respectively.
\end{abstract}

Keywords: tight focusing, radially polarized, circular partially coherent, optical needle, dark channel.

\section{Introduction}

Beams with desired intensity distribution have attracted much attention. One example is an optical needle, i.e., a focal pattern with a small beam size and long depth of focus, which is of great significance in many applications such as optical data storage, photolithography, and super-resolution microscopy [1- $\underline{-6}$ ]. Another example is optical bottle beams, i.e., beams with a dark center surrounded by three-dimensional regions of higher intensity, which can be applied in trapping particles with refractive indices lower than that of the surrounding medium [7]. Due to the special intensity distribution and important applications, it is an attractive topic for structuring the optical needle and

\footnotetext{
${ }^{\dagger}$ These authors contributed equally to this work.
} 
optical bottle beams, and a variety of methods have been proposed for generating such beams $[\underline{6}-\underline{20}]$. However, the beams are limited to completely coherent laser beams in most of the studies. In view of the universality and significance of partially coherent beams, it would be a valuable work to explore the generation of sub-wavelength optical needle and optical bottle beams adopting partially coherent beams.

Recently, SANTARSIERO and his collaborators proposed a new class of a partially coherent light source, namely a circular partially coherent light source [21, 22]. This type of a partially coherent beam exhibits perfect coherence along any annulus, while the coherence between two points with different radial distances is partial or even vanishing [21-24]. In this study, we investigate the focusing of a radially polarized circular partially coherent (RPCPC) beam by a high numerical aperture (NA) objective. The study shows that a super-long optical needle with longitudinal polarization and a super-long dark channel with transversal polarization can be obtained by a tightly focused RPCPC beam. Moreover, the full width at half maximum (FWHM) of the optical needle and the dark channel of the optical bottle beams are both sub-wavelength.

\section{Theoretical analysis}

The theory of tight focusing of light beams was described by RicHARDS and WoLF originally [25]. According to the theory, the electric field near the focus formed by a tightly focused radially polarized completely coherent laser beam can be expressed as [26]

$$
\begin{array}{r}
\mathbf{E}(\rho, \psi, z)=\frac{i E_{0}}{\lambda} \int_{0}^{\alpha} \int_{0}^{2 \pi} \exp [-i k z \cos \theta-i k \rho \sin \theta \cos (\psi-\varphi)] \\
\times P(\varphi, \theta) \mathbf{K}(\varphi, \theta) \sin \theta \sqrt{\cos \theta} \mathrm{d} \varphi \mathrm{d} \theta
\end{array}
$$

where $\theta$ is the angle of convergence, $\varphi$ is the polar angle of incident plane, $\lambda$ is the wavelength of incident beam, $k=2 \pi / \lambda$ is the wave-number, $\alpha=\sin ^{-1}(\mathrm{NA})$ is the maximal angle determined by the NA of the objective, and $E_{0}$ is the characteristic amplitude of incident beam. Variables $\rho, \psi$ and $z$ are the cylindrical coordinates of an observation point near the focus. $P(\varphi, \theta)$ is the pupil apodization function at the exit pupil, and

$$
\mathbf{K}(\varphi, \theta)=\left[\begin{array}{l}
\cos \theta \cos \varphi \mathbf{e}_{x} \\
\cos \theta \sin \varphi \mathbf{e}_{y} \\
\sin \theta \mathbf{e}_{z}
\end{array}\right]
$$

where $\mathbf{e}_{x}, \mathbf{e}_{y}$, and $\mathbf{e}_{z}$ are the unit vectors in the $x, y$ and $z$ directions, respectively. Clearly, a tightly focused radially polarized beam is depolarized into three components, i.e., $x$-, $y$-, and $z$-polarization components in Cartesian coordinates.

For a partially coherent illumination, the second order correlation properties of the field can be characterized by the cross-spectral density (CSD). In Cartesian coordinates, the second-order correlation properties of the beam near the focal region can be 
characterized by a $3 \times 3$ electric cross-spectral density matrix $\mathbf{W}\left(\boldsymbol{\rho}_{1}, \boldsymbol{\rho}_{2}, z\right)$. The elements of the $3 \times 3$ matrix are given by [27]

$$
\begin{aligned}
W_{i j}\left(\boldsymbol{\rho}_{1}, \boldsymbol{\rho}_{2}, z\right)= & W_{i j}\left(\rho_{1}, \rho_{2}, \psi_{1}, \psi_{2}, z\right)=\left\langle E_{i}\left(\rho_{1}, \psi_{1}, z\right) E_{j}^{*}\left(\rho_{2}, \psi_{2}, z\right)\right\rangle \\
= & \left(\frac{E_{0}}{\lambda}\right)^{2} \int_{0}^{\alpha} \int_{0}^{\alpha} \int_{0}^{2 \pi} \int_{0}^{2 \pi} P\left(\varphi_{1}, \theta_{1}\right) P\left(\varphi_{2}, \theta_{2}\right) \mathbf{K}_{i}\left(\varphi_{1}, \theta_{1}\right) \mathbf{K}_{j}^{\mathrm{T}}\left(\varphi_{2}, \theta_{2}\right) \\
& \times g\left(\varphi_{1}, \theta_{1}, \varphi_{2}, \theta_{2}\right) \exp \left[-i k z \cos \theta_{1}-i k \rho_{1} \sin \theta_{1} \cos \left(\psi_{1}-\varphi_{1}\right)\right] \\
& \times \sin \theta_{1} \sin \theta_{2} \exp \left[i k z \cos \theta_{2}+i k \rho_{2} \sin \theta_{2} \cos \left(\psi_{2}-\varphi_{2}\right)\right] \\
& \times \sqrt{\cos \theta_{1}} \sqrt{\cos \theta_{2}} \mathrm{~d} \varphi_{1} \mathrm{~d} \varphi_{2} \mathrm{~d} \theta_{1} \mathrm{~d} \theta_{2}, \quad(i, j=x, y, z)
\end{aligned}
$$

where $g\left(\varphi_{1}, \theta_{1}, \varphi_{2}, \theta_{2}\right)$ is the two-point pupil apodization correlator of the incident beam. It should be noted that the superscript $\mathrm{T}$ denotes the transpose of the matrix of $\mathbf{K}(\varphi, \theta)$ indicating that $\mathbf{K}^{\mathrm{T}}(\varphi, \theta)$ is a $1 \times 3$ matrix. In this paper, RPCPC beam is taken as an incident laser beam, and its cross-spectral density (CSD) can be expressed as

$$
\begin{aligned}
& W_{0}\left(\mathbf{r}_{1}, \mathbf{r}_{2}\right)=\frac{E_{0}^{2}}{\omega_{0}^{2}} \exp \left(-\frac{r_{1}^{2}+r_{2}^{2}}{\omega_{0}^{2}}\right)\left[\begin{array}{ll}
\operatorname{sinc}\left(\frac{r_{2}^{2}-r_{1}^{2}}{\delta^{2}}\right) x_{1} x_{2} & \operatorname{sinc}\left(\frac{r_{2}^{2}-r_{1}^{2}}{\delta^{2}}\right) x_{1} y_{2} \\
\operatorname{sinc}\left(\frac{r_{2}^{2}-r_{1}^{2}}{\delta^{2}}\right) y_{1} x_{2} & \operatorname{sinc}\left(\frac{r_{2}^{2}-r_{1}^{2}}{\delta^{2}}\right) y_{1} y_{2}
\end{array}\right] \\
& =\frac{E_{0}^{2}}{\omega_{0}^{2}} \exp \left(-\frac{r_{1}^{2}+r_{2}^{2}}{\omega_{0}^{2}}\right) \operatorname{sinc}\left(\frac{r_{2}^{2}-r_{1}^{2}}{\delta^{2}}\right)\left[\begin{array}{ll}
x_{1} x_{2} & x_{1} y_{2} \\
y_{1} x_{2} & y_{1} y_{2}
\end{array}\right]
\end{aligned}
$$

where $\operatorname{sinc}(x)=\sin (\pi x) / \pi x, \omega_{0}$ is the beam width, $\delta$ is the initial coherent length, and $\mathbf{r}=(r, \varphi)$ is the position vector at the source plane. The variables $r$ are position vectors on the left hand side of Eq. (4), but they are scalars on the right hand side because the cross-spectral density of circular partially coherent Gaussian (CPCG) beam is angle-independent. Therefore, the correlation between any two of the parallel or orthometric electric field components of the incident RPCPC beam is [27]

$$
\mu_{i j}\left(r_{1}, r_{2}\right)=\frac{W_{i j}\left(r_{1}, r_{2}\right)}{\sqrt{W_{i j}\left(r_{1}, r_{1}\right) W_{i j}\left(r_{2}, r_{2}\right)}}=\operatorname{sinc}\left(\frac{r_{2}^{2}-r_{1}^{2}}{\delta^{2}}\right), \quad(i, j=x, y)
$$

The coordinate system is shown in Fig. 1a. Figure $1 \mathbf{b}$ illustrate the intensity distribution of incident RPCPC beam, and the direction of polarization is denoted by the 


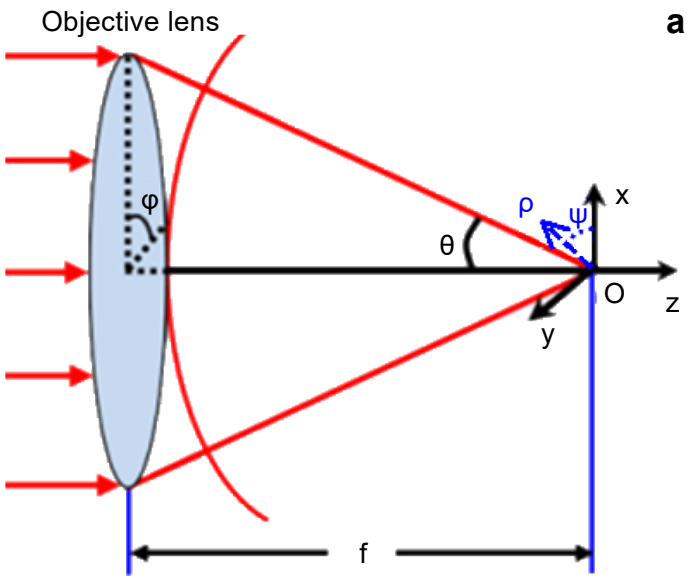

Incident RPCPC beam
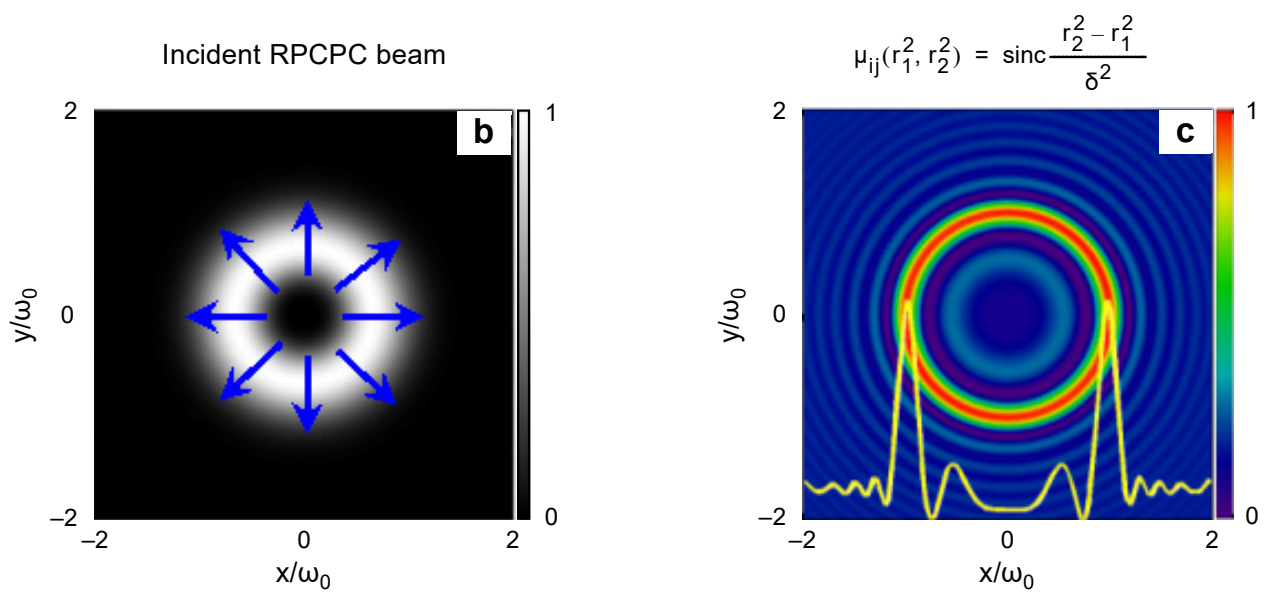

Fig. 1. Tight focusing system (a). The intensity distribution of incident RPCPC beam (b). The initial coherence of incident RPCPC beam (c). The parameters of (a) and (b) are chosen as $\lambda=633 \mathrm{~nm}, \omega_{0}=5 \mathrm{~mm}$, and $\delta=0.3 \omega_{0}$.

blue arrows. By setting the first position $r_{1}=\omega_{0}$, and $\delta=0.3 \omega_{0}$ the distribution of the initial coherence of the incident RPCPC beam is shown in Fig. 1c.

Under the sine condition [25], i.e., $r=f \sin \theta$, where $f$ is the focal length of the objective, the two point pupil apodizaiton correlation function of the incident beam can be expressed as

$$
\begin{aligned}
F\left(\varphi_{1}, \varphi_{2}, \theta_{1}, \theta_{2}\right) & =P\left(\varphi_{1}, \theta_{1}\right) P\left(\varphi_{2}, \theta_{2}\right) g\left(\varphi_{1}, \theta_{1}, \varphi_{2}, \theta_{2}\right) \\
& =\exp \left[\frac{-f^{2}\left(\sin ^{2} \theta_{1}+\sin ^{2} \theta_{2}\right)}{\omega_{0}^{2}}\right] \operatorname{sinc}\left(\frac{f^{2} \sin ^{2} \theta_{2}-f^{2} \sin ^{2} \theta_{1}}{\delta^{2}}\right)
\end{aligned}
$$

Substituting Eq. (6) into Eq. (3), and after same tedious integration, the elements $\mathbf{W}\left(\boldsymbol{\rho}_{1}, \boldsymbol{\rho}_{2}, z\right)$ in the focal region can be obtained as follows: 


$$
\begin{aligned}
& W_{i j}\left(\rho_{1}, \rho_{2}, \psi_{1}, \psi_{2}, z\right)=4\left(\frac{E_{0} \pi}{\lambda}\right)^{2} \int_{0}^{\alpha} \int_{0}^{\alpha} \frac{f^{2}}{\omega_{0}^{2}} \cos ^{3 / 2} \theta_{1} \cos ^{3 / 2} \theta_{2} \\
& \times \exp \left[\frac{-f^{2}\left(\sin ^{2} \theta_{1}+\sin ^{2} \theta_{2}\right)}{\omega_{0}^{2}}\right] \operatorname{sinc}\left(\frac{f^{2} \sin ^{2} \theta_{2}-f^{2} \sin ^{2} \theta_{1}}{\delta^{2}}\right) \\
& \quad \times \exp \left[i k z\left(\cos \theta_{2}-\cos \theta_{1}\right)\right] M_{i j}\left(\rho_{1}, \rho_{2}, \psi_{1}, \psi_{2}, \theta_{1}, \theta_{2}\right) \mathrm{d} \theta_{1} \mathrm{~d} \theta_{2}, \quad(i, j=x, y, z)
\end{aligned}
$$

where $M_{i j}\left(\rho_{1}, \rho_{2}, \psi_{1}, \psi_{2}, \theta_{1}, \theta_{2}\right)$, for the elements of $\mathbf{W}\left(\boldsymbol{\rho}_{1}, \boldsymbol{\rho}_{2}, z\right)$ are

$$
M_{x x}\left(\rho_{1}, \rho_{2}, \psi_{1}, \psi_{2}, \theta_{1}, \theta_{2}\right)=J_{1}\left(k \rho_{1} \sin \theta_{1}\right) J_{1}\left(k \rho_{2} \sin \theta_{2}\right) \sin ^{2} \theta_{1} \sin ^{2} \theta_{2} \cos \psi_{1} \cos \psi_{2}
$$

$M_{x y}\left(\rho_{1}, \rho_{2}, \psi_{1}, \psi_{2}, \theta_{1}, \theta_{2}\right)=J_{1}\left(k \rho_{1} \sin \theta_{1}\right) J_{1}\left(k \rho_{2} \sin \theta_{2}\right) \sin ^{2} \theta_{1} \sin ^{2} \theta_{2} \cos \psi_{1} \sin \psi_{2}$

$M_{x z}\left(\rho_{1}, \rho_{2}, \psi_{1}, \psi_{2}, \theta_{1}, \theta_{2}\right)=-i J_{1}\left(k \rho_{1} \sin \theta_{1}\right) J_{0}\left(k \rho_{2} \sin \theta_{2}\right) \sin ^{2} \theta_{1} \sin ^{3} \theta_{2} \cos \psi_{1}$

$M_{y x}\left(\rho_{1}, \rho_{2}, \psi_{1}, \psi_{2}, \theta_{1}, \theta_{2}\right)=J_{1}\left(k \rho_{1} \sin \theta_{1}\right) J_{1}\left(k \rho_{2} \sin \theta_{2}\right) \sin ^{2} \theta_{1} \sin ^{2} \theta_{2} \cos \psi_{2} \sin \psi_{1}$

$M_{y y}\left(\rho_{1}, \rho_{2}, \psi_{1}, \psi_{2}, \theta_{1}, \theta_{2}\right)=J_{1}\left(k \rho_{1} \sin \theta_{1}\right) J_{1}\left(k \rho_{2} \sin \theta_{2}\right) \sin ^{2} \theta_{1} \sin ^{2} \theta_{2} \sin \psi_{1} \sin \psi_{2}$

$M_{y z}\left(\rho_{1}, \rho_{2}, \psi_{1}, \psi_{2}, \theta_{1}, \theta_{2}\right)=-i J_{1}\left(k \rho_{1} \sin \theta_{1}\right) J_{0}\left(k \rho_{2} \sin \theta_{2}\right) \sin ^{2} \theta_{1} \sin ^{3} \theta_{2} \sin \psi_{1}$

$M_{z x}\left(\rho_{1}, \rho_{2}, \psi_{1}, \psi_{2}, \theta_{1}, \theta_{2}\right)=-i J_{1}\left(k \rho_{2} \sin \theta_{2}\right) J_{0}\left(k \rho_{1} \sin \theta_{1}\right) \sin ^{2} \theta_{2} \sin ^{3} \theta_{1} \cos \psi_{2}$

$M_{z y}\left(\rho_{1}, \rho_{2}, \psi_{1}, \psi_{2}, \theta_{1}, \theta_{2}\right)=-i J_{1}\left(k \rho_{2} \sin \theta_{2}\right) J_{0}\left(k \rho_{1} \sin \theta_{1}\right) \sin ^{2} \theta_{2} \sin ^{3} \theta_{1} \sin \psi_{2}$

$M_{z z}\left(\rho_{1}, \rho_{2}, \psi_{1}, \psi_{2}, \theta_{1}, \theta_{2}\right)=J_{0}\left(k \rho_{1} \sin \theta_{1}\right) J_{0}\left(k \rho_{2} \sin \theta_{2}\right) \sin ^{3} \theta_{1} \sin ^{3} \theta_{2} \sin \psi_{1}$

In equations (8) through (16), $J_{0}$ and $J_{1}$ are the zero-order and first-order Bessel functions of the first kind, respectively. Setting $\rho_{1}=\rho_{2}$ and $\psi_{1}=\psi_{2}$, the intensity of $x$-polarization component, $y$-polarization component, $z$-polarization component and total intensity distribution in the focal region can be expressed as 


$$
\begin{gathered}
I_{x}\left(\rho_{1}, \rho_{1}, \psi_{1}, \psi_{1}, z\right)=W_{x x}\left(\rho_{1}, \rho_{1}, \psi_{1}, \psi_{1}, z\right) \\
I_{y}\left(\rho_{1}, \rho_{1}, \psi_{1}, \psi_{1}, z\right)=W_{y y}\left(\rho_{1}, \rho_{1}, \psi_{1}, \psi_{1}, z\right) \\
I_{z}\left(\rho_{1}, \rho_{1}, \psi_{1}, \psi_{1}, z\right)=W_{z z}\left(\rho_{1}, \rho_{1}, \psi_{1}, \psi_{1}, z\right) \\
I_{\text {total }}\left(\rho_{1}, \rho_{1}, \psi_{1}, \psi_{1}, z\right)=I_{x}\left(\rho_{1}, \rho_{1}, \psi_{1}, \psi_{1}, z\right)+I_{y}\left(\rho_{1}, \rho_{1}, \psi_{1}, \psi_{1}, z\right) \\
+I_{z}\left(\rho_{1}, \rho_{1}, \psi_{1}, \psi_{1}, z\right)
\end{gathered}
$$

In addition, the intensity of transverse polarized component can be expressed as

$$
I_{\text {transverse }}\left(\rho_{1}, \rho_{1}, \psi_{1}, \psi_{1}, z\right)=W_{x x}\left(\rho_{1}, \rho_{1}, \psi_{1}, \psi_{1}, z\right)+W_{y y}\left(\rho_{1}, \rho_{1}, \psi_{1}, \psi_{1}, z\right)
$$

Moreover, the expression of Eq. (19) is the intensity of a longitudinal polarized component.

\section{Results and discussions}

Intensity distribution of different polarized components of the RPCPC beam near the focus can be calculated by Eqs. (17)-(21). The total intensity and its components in
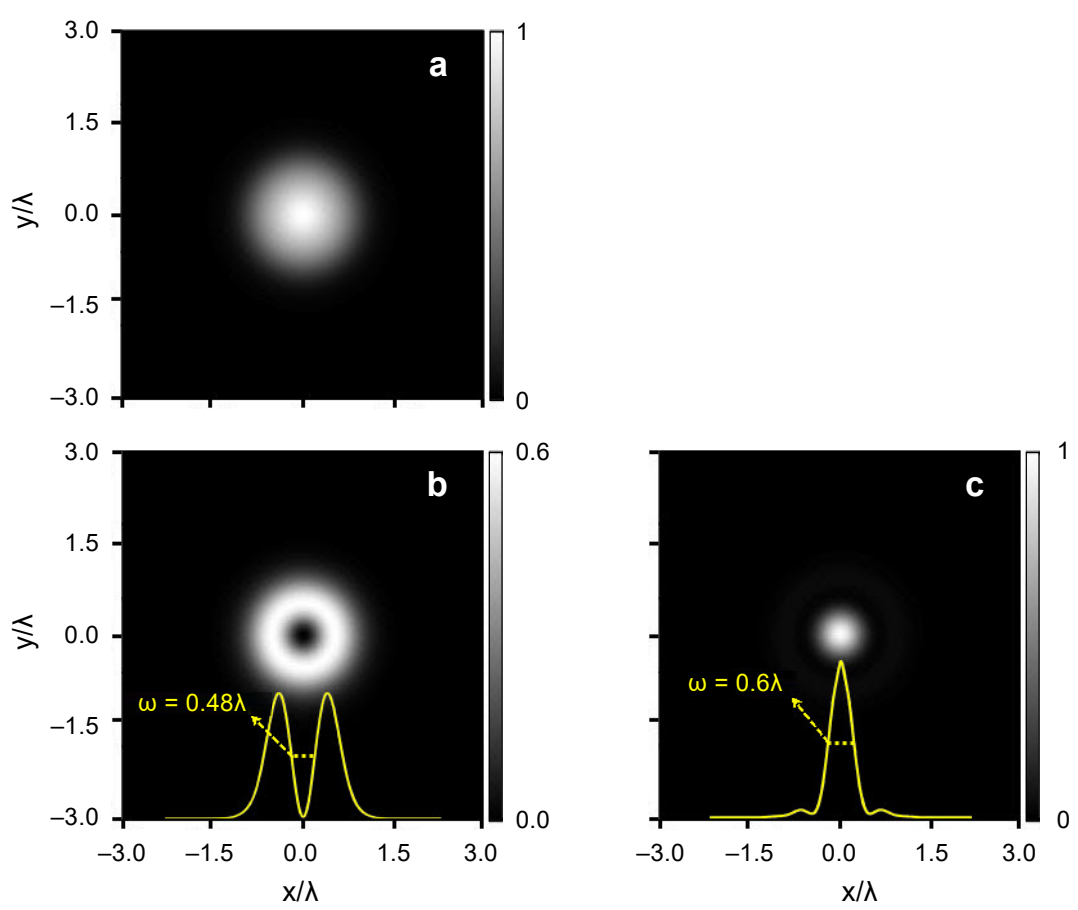

Fig. 2. Normalized intensity distribution of RPCPC beam in focal plane. Total intensity (a), transverse polarized component (b), and longitudinal polarized component (c). The parameters for calculation are chosen as $\lambda=633 \mathrm{~nm}, \omega_{0}=5 \mathrm{~mm}, \mathrm{NA}=0.9, f=1 \mathrm{~cm}, E_{0}=1$, and $\delta=\omega_{0}$. 
the focal plane of the objective are shown in Fig. 2. The transverse component of the intensity in the focal plane is annular-shaped, and the FWHM of the dark hollow is $0.48 \lambda$. The longitudinal component of the intensity is quasi-Gaussian-shaped with FWHM of $0.6 \lambda$.

Figure 2 shows that intensity distribution of RPCPC beam in the focal plane is circularly symmetric. Therefore, we can consider only the radial direction to study the influence of initial coherent length on intensity distribution. The intensity profiles of focused RPCPC beam with different initial coherent length are presented in Fig. 3. Although the spot size of the total intensity increases gradually with the decrease in initial coherent length, the lateral dimension of the dark hollow of the transverse component and the focal spot of longitudinal component keep nearly invariant.

In addition to the intensity of the focal plane, we have also been interested in the intensity distribution of whole focal pattern. Figure 4 indicates that the focal patterns lengthen along the longitudinal direction with the decrease in the initial coherent length of the incident RPCPC beam. The focal pattern is like a light pillar with the initial coherent length of $0.3 \omega_{0}$ (Fig. 4c). With the decrement of the initial coherent length, the transverse component of intensity evolves into dark channel, which is a non-diffracting
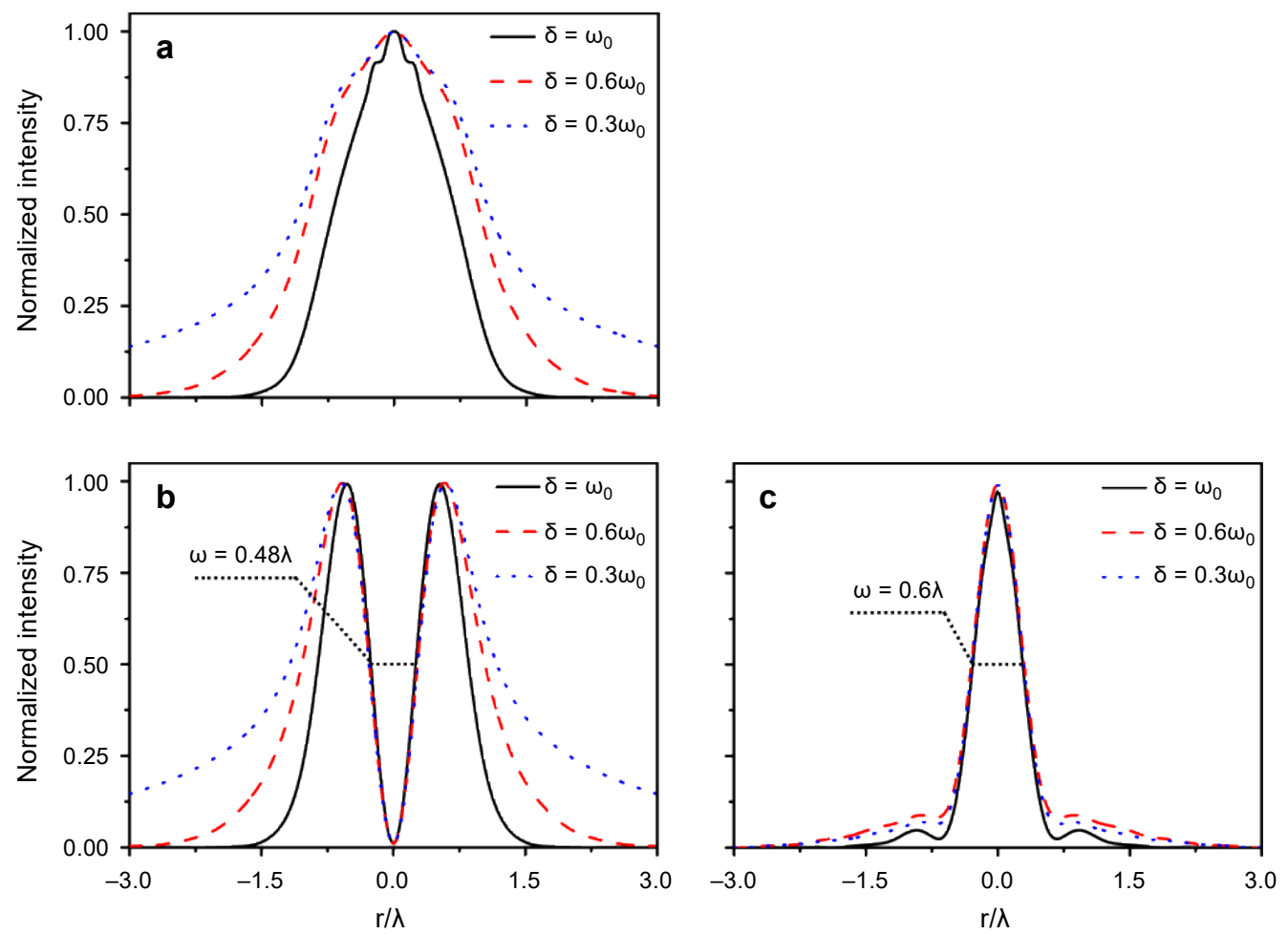

Fig. 3. Normalized intensity distribution curves of RPCPC beam for different $\delta$ in the focal plane. Total intensity (a), transverse polarized component (b), and longitudinal polarized component (c). The parameters for calculation are chosen as $\lambda=633 \mathrm{~nm}, \omega_{0}=5 \mathrm{~mm}, \mathrm{NA}=0.9, f=1 \mathrm{~cm}$, and $E_{0}=1$. 

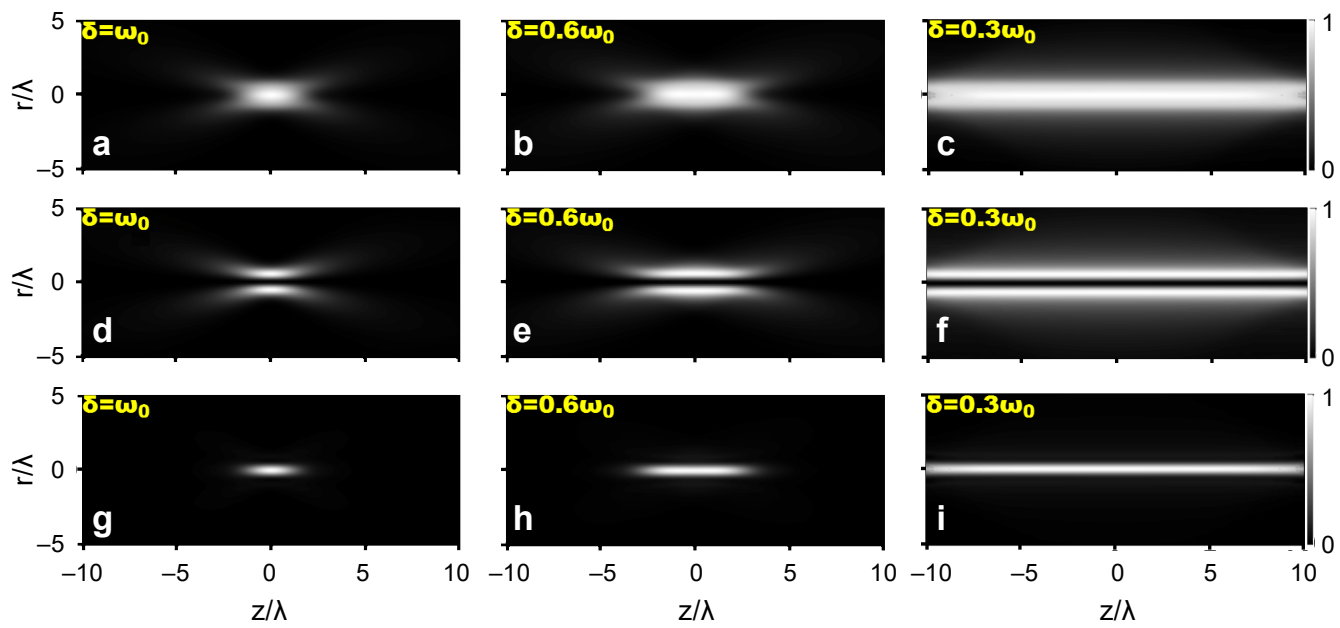

Fig. 4. Intensity distributions of total intensity $(\mathbf{a}-\mathbf{c})$, transverse polarized component $(\mathbf{d}-\mathbf{f})$ and longitudinal polarized component $(\mathbf{g}-\mathbf{i})$ of RPCPC beam in $r-z$ plane near the focus for different $\delta$. The parameters for calculation are chosen as $\lambda=633 \mathrm{~nm}, \omega_{0}=5 \mathrm{~mm}, \mathrm{NA}=0.9, f=1 \mathrm{~cm}$, and $E_{0}=1$.

focal hole surrounded in the radial direction by the regions of higher intensity as shown in Fig. 4f. Meanwhile, the longitudinal component of intensity evolves into the optical needle, which is a focal pattern with a small beam size and long depth of focus as shown in Fig. 4i. Furthermore, it is interesting to find that the lateral dimension of the dark channel and optical needle remains nearly intact during the evolution. This indicates that we can engineer the source coherent length of the incident RPCPC beam to modulate the length of a dark channel and optical needle, meanwhile keeping the lateral

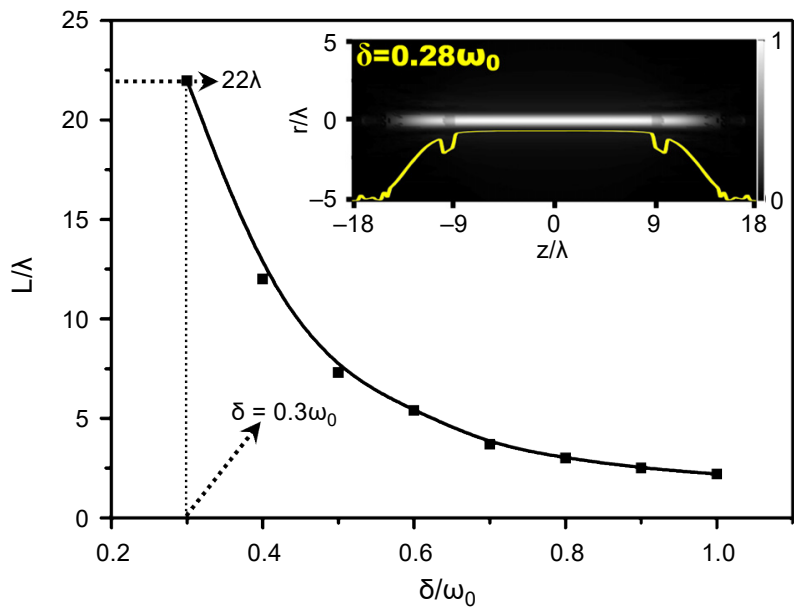

Fig. 5. Length of the optical needle as a function of initial coherent length. Inset is the intensity distribution of longitudinal polarized component in $r-z$ plane for $\delta=0.28 \omega_{0}$. The parameters for calculation are chosen as $\lambda=633 \mathrm{~nm}, \omega_{0}=5 \mathrm{~mm}, \mathrm{NA}=0.9, f=1 \mathrm{~cm}$, and $E_{0}=1$. 
dimension invariant. This length-adjustable dark channel and optical needle may have a potential application in optical data storage, photolithography, super-resolution microscopy, and particles trapping.

Figure 5 illustrates the relation between the length of the optical needle and initial coherent length of the incident RPCPC beam. The length of optical needle reduces gradually with increasing initial coherent length. The maximal length of the optical needle is $22 \lambda$, which is generated by a beam with the initial coherent length of $0.3 \omega_{0}$. Although the length of the focal pattern can be further increased with decreasing initial coherent length, the needle shape is non-uniform, as shown in the inset of Fig. 5.

Now we focus on the influence of numerical aperture (NA) of the objective on the intensity distribution in the focal region. As mentioned above, we also consider only the $r$ direction to study the influence of NA on the intensity distribution, because the intensity distribution is circularly symmetric. Intensity distribution with Gaussian profile is generated with NA of 0.85 and 0.99 , while a relative dark core is observed with NA of 0.7 (Fig. 6a). Figures $6 \mathbf{b}$ and $6 \mathbf{c}$ show the intensity distribution curves of the
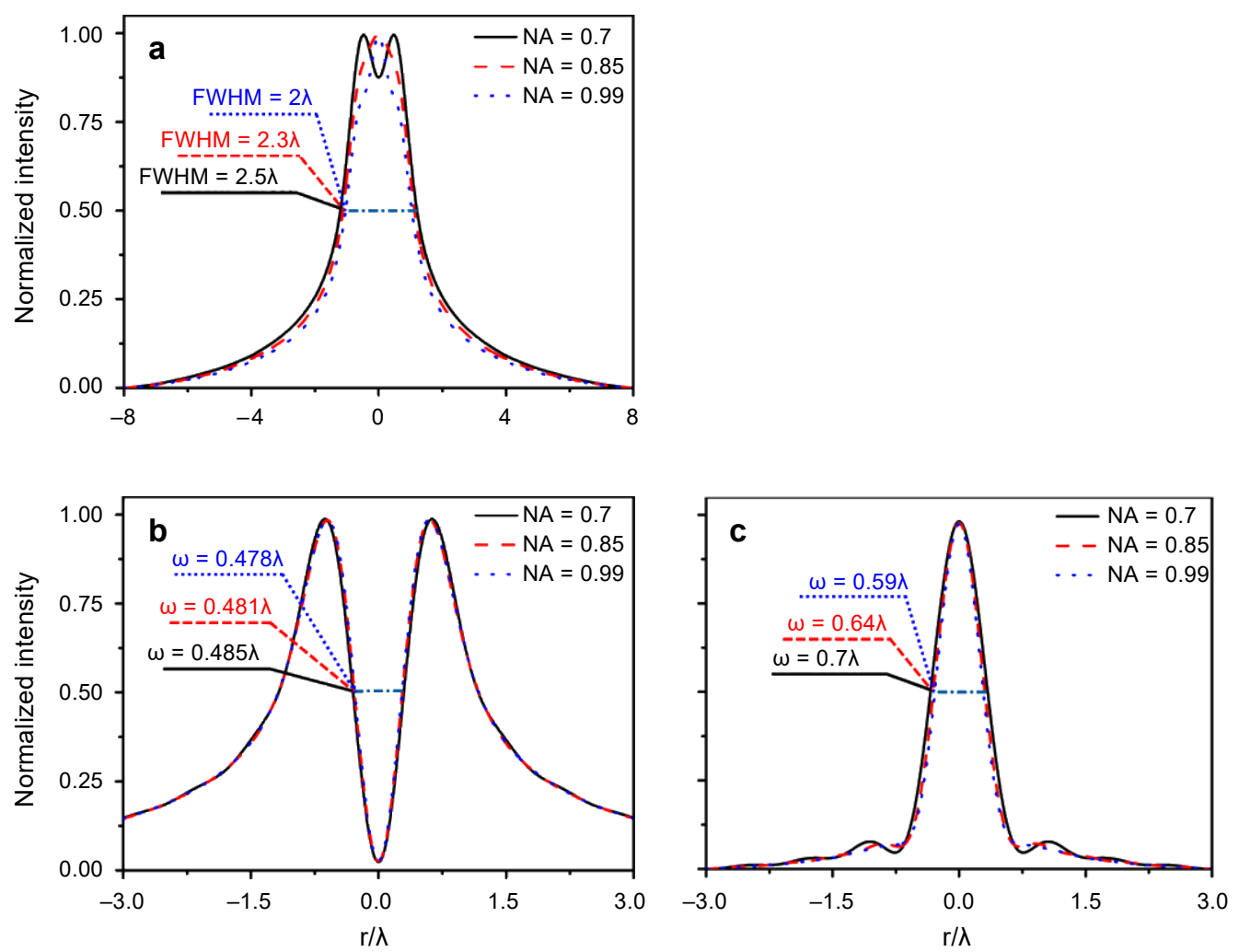

Fig. 6. Intensity distribution curve of the total intensity for different NA (a). Intensity distribution curve of the transverse polarized component for different NA (b). Intensity distribution curve of longitudinal polarized component for different NA (c). The parameters for calculation are chosen as $\lambda=633 \mathrm{~nm}$, $\omega_{0}=5 \mathrm{~mm}, \delta=0.3 \omega_{0}, f=1 \mathrm{~cm}$, and $E_{0}=1$. 


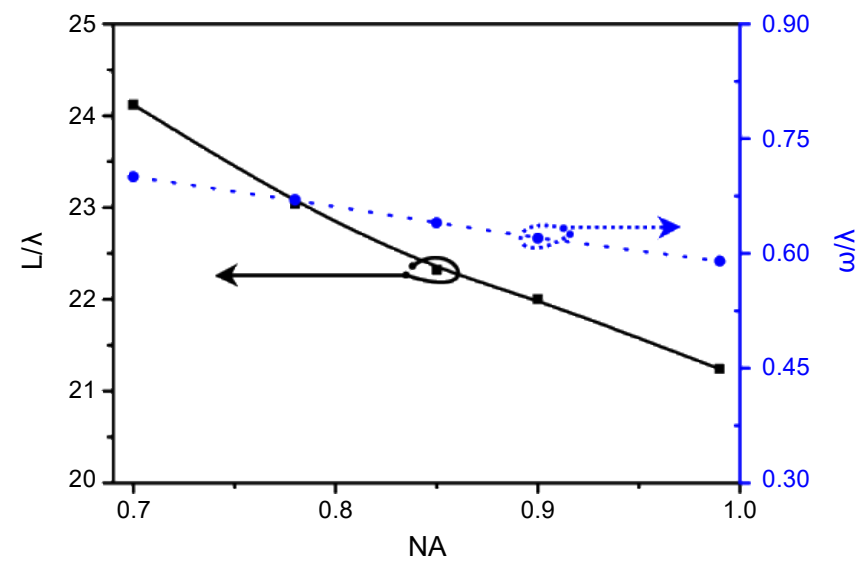

Fig. 7. The length and the FWHM of the optical needle as a function of NA of the objective. The parameters for calculation are chosen as $\lambda=633 \mathrm{~nm}, \omega_{0}=5 \mathrm{~mm}, \delta=0.3 \omega_{0}, f=1 \mathrm{~cm}$, and $E_{0}=1$.

transverse polarized component and the longitudinal polarized component. The lateral dimension of the dark channel and the optical needle decrease gradually with increasing NA of the objective. Nevertheless, it should be noted that the reduction of the lateral dimension is relatively small. Figure 7 shows that the length and FWHM of the optical needle decrease with the increment of NA. This is because a smaller focal pattern can be produced with a larger NA of the objective.

\section{Concluding remarks}

We theoretically investigate the tight focusing the RPCPC beams through a high NA objective. The results demonstrated that the sub-wavelength optical needle with longitudinal polarization, and the dark channel with transverse polarized can be generated in the focal region. Moreover, the length of the optical needle and dark channel can be adjusted by controlling the coherent length of the incident RPCPC beam, and the length adjustment would not induce the unwilling expansion of the lateral dimension. The obtained maximum length of the optical needle and the dark channel is about $22 \lambda$, and the FWHM of the dark channel and the optical needle are $0.48 \lambda$ and $0.6 \lambda$, respectively. And the length and FWHM of the optical needle are influenced by the NA of the objective.

Acknowledgements - This research was supported by National Natural Science Foundation of China (NSFC) under grant numbers 61575070, 11750110426, Natural Science Foundation of Fujian province under grant number 2018J01551, Natural Science Foundation of the Fujian Higher Education Institutions (JZ160452, JAT160293, JAT160457, FBJG20180015, IRTSTFJ), and Zhangzhou Natural Science Foundation (ZZ2016J40, ZZ2019J01). 


\section{References}

[1] Wang H., Shi L., Lukyanchuk B., Sheppard C., Chong C., Creation of a needle of longitudinally povolarized light in vacuum using binary optics, Nature Photonics 2, 2008, pp. 501-505, DOI: 10.1038 / nphoton.2008.127.

[2] Scott T.F., Kowalski B.A., Sullivan A.C., Bowman C.N., McLeod R.R., Two-color single-photon photoinitiation and photoinhibition for subdiffraction photolithography, Science 324(5929), 2009, pp. 913-917, DOI: 10.1126/science.1167610.

[3] Yang J., Gong L., Shen Y., Wang L.V., Synthetic Bessel light needle for extended depth-of-field microscopy, Applied Physics Letters 113(18), 2018, article 181104, DOI: 10.1063/1.5058163.

[4] Chen Z., Hu X., JI X., Pu J., Needle beam generated by a laser beam passing through a scattering medium, IEEE Photonics Journal 10(5), 2018, article 6501108, DOI: 10.1109/JPHOT.2018.2871216.

[5] Man Z., Min C., Du L., Zhang Y., Zhu S., Yuan X., Sub-wavelength sized transversely polarized optical needle with exceptionally suppressed side-lobes, Optics Express 24(2), 2016, pp. 874-882, DOI: $10.1364 / \mathrm{OE} .24 .000874$.

[6] Hu K., Chen Z., Pu J., Generation of super-length optical needle by focusing hybridly polarized vector beams through a dielectric interface, Optics Letters 37(16), 2012, pp. 3303-3305, DOI: 10.1364/OL.37.003303.

[7] Lalithambigai K., Suresh P., Ravi V., Prabakaran K., Jaroszewicz Z., Rajesh K.B., Anbarasan P.M., Pillai T.V.S., Generation of sub wavelength super-long dark channel using high NA lens axicon, Optics Letters 37(6), 2012, pp. 999-1001, DOI: 10.1364/OL.37.000999.

[8] Liu Y., Zhang Z., Li C., Liu D., KuANG C., Rotating light fields of an azimuthally polarized light beam generated by two-belt spiral phase modulation, Journal of Modern Optics 65(20), 2018, pp. 2295-2300, DOI: $10.1080 / 09500340.2018 .1506056$.

[9] Zhou G., Ji Z., Zhou Y., Chen R., Focusing of radially polarized Lorentz-Gauss beams with the power-exponent-phase vortex, Journal of Modern Optics 65(7), 2018, pp. 796-802, DOI: 10.1080 / 09500340.2017 .1401134$.

[10] Xu H., Zhang W., Qu J., HuAng W., Improving the trapping capability using radially polarized narrow-width annular beam, Journal of Modern Optics 63(5), 2016, pp. 495-500, DOI: 10.1080 / 09500340.2014 .922634$.

[11] Weng X., Song Q., Li X., Gao X., Guo H., Qu J., Zhuang S., Free-space creation of ultralong anti -diffracting beam with multiple energy oscillations adjusted using optical pen, Nature Communications 9, 2018, article 5035, DOI: 10.1038/s41467-018-07282-y.

[12] Maucher F., Skupin S., Gardiner S.A., Hughes I.G., Creating complex optical longitudinal polarization structures, Physical Review Letters 120(16), 2018, article 163903, DOI: 10.1103/PhysRevLett. 120.163903.

[13] Liu S., Qi S., Zhang Y., Li P., Wu D., Han L., Zhao J., Highly efficient generation of arbitrary vector beams with tunable polarization, phase, and amplitude, Photonics Research 6(4), 2018, pp. 228-233, DOI: 10.1364/PRJ.6.000228.

[14] Drevinskas R., Zhang Y., Beresna M., Gecevicius M., Kazanskit A.G., Svirko Y.P., Kazansky P.G., Laser material processing with tightly focused cylindrical vector beams, Applied Physics Letters 108(22), 2016, article 221107, DOI: 10.1063/1.4953455.

[15] Tang M., Zhao D., Li X., Li H., Focusing properties of radially polarized multi-cosine Gaussian correlated Schell-model beams, Optics Communications 396, 2017, pp. 249-256, DOI: 10.1016 / j.optcom.2017.03.063.

[16] Zhang P., Li T., Zhu J., Zhu X., Yang S., Wang Y., Yin X., Zhang X., Generation of acoustic self -bending and bottle beams by phase engineering, Nature Communications 5, 2014, article 4316, DOI: $10.1038 /$ ncomms5316.

[17] Epstein I., ARIE A., Dynamic generation of plasmonic bottle-beams with controlled shape, Optics Letters 39(11), 2014, pp. 3165-3168, DOI: 10.1364/OL.39.003165. 
[18] Xu H., Zhang Z., Qu J., Huang W., The tight focusing properties of Laguerre-Gaussian correlated Schell-modle beams, Journal of Modern Optics 63(15), 2016, pp. 1429-1437, DOI: 10.1080/ 09500340.2016 .1151565$.

[19] LiU S., You S., FAng Y., WANG Y., KuANG C., LiU X., Effects of polarization and phase modulation on the focal spot in 4Pi microscopy, Journal of Modern Optics 63(12), 2016, pp. 1145-1157, DOI: $10.1080 / 09500340.2015 .1129077$.

[20] Zhang Z., Xu H., Qu J., HuAng W., Radiation forces of highly focused radially polarized hollow sinh-Gaussian beams on a Rayleigh metallic particle, Journal of Modern Optics 62(9), 2015, pp. 754-760, DOI: 10.1080/09500340.2015.1005188.

[21] Santarsiero M., Martínez-Herrero R., Maluenda D., de Sande J.C.G., Piquero G., Gori F., Partially coherent sources with circular coherence, Optics Letters 42(8), 2017, pp. 1512-1515, DOI: 10.1364/OL.42.001512.

[22] Santarsiero M., Martínez-Herrero R., Maluenda D., de Sande J.C.G., Piquero G., Gori F., Synthesis of circularly coherent sources, Optics Letters 42(20), 2017, pp. 4115-4118, DOI: $10.1364 /$ OL.42.004115.

[23] Ding C., Koivurova M., Turunen J., PAn L., Self-focusing of a partially coherent beam with circular coherence, Journal of the Optical Society of America A 34(8), 2017, pp. 1441-1447, DOI: $10.1364 /$ JOSAA.34.001441.

[24] Lin H., Zhou X., Chen Z., Sasaki O., Li Y., Pu J., Tight focusing properties of a circular partially coherent Gaussian beam, Journal of the Optical Society of America A 35(12), 2018, pp. 1974-1980, DOI: 10.1364/JOSAA.35.001974.

[25] Richards B., Wolf E., Electromagnetic diffraction in optical systems. II. Structure of the image field in an aplanatic system, Proceedings of the Royal Society A 253(1274), 1959, pp. 358-379, DOI: 10.1098/rspa.1959.0200.

[26] Gu M., Advanced Optical Imaging Theory, Springer, Heidelberg, 1999.

[27] Lindfors K., Setälä T., Kaivola M., Friberg A.T., Degree of polarization in tightly focused optical fields, Journal of the Optical Society of America A 22(3), 2005, pp. 561-568, DOI: 10.1364/ JOSAA.22.000561.

Received April 17, 2019

in revised form May 28, 2019 